\title{
Proximity Structures for Geometric Graphs
}

\author{
Sanjiv Kapoor and Xiang-Yang Li \\ Department of Computer Science, Illinois Institute of Technology \\ 10 W. 31st Street, Chicago, IL 60616, USA \\ kapoor@iit.edu,xli@cs.iit.edu
}

\begin{abstract}
In this paper we study proximity structures like Delaunay triangulations based on geometric graphs, i.e. graphs which are subgraphs of the complete geometric graph. Given an arbitrary geometric graph $G$, we define several restricted Voronoi diagrams, restricted Delaunay triangulations, relative neighborhood graphs, Gabriel graphs and then study their complexities when $G$ is a general geometric graph or $G$ is some special graph derived from the application area of wireless networks. Besides being of fundamental interest these structures have applications in topology control for wireless networks.
\end{abstract}

\section{Introduction}

Given a set $S$ of two dimensional points, many geometric proximity structures were defined for various applications, such as the Delaunay triangulation [1$3]$, the Voronoi Diagram [2,3], the Gabriel graph (GG) [4,5], and the relative neighborhood graph (RNG) [6-8]. These diagrams are defined with respect to a geometric neighborhood. For example an edge $u v$ is in GG if and only if the circle with $u v$ as a diameter, denoted by $\operatorname{disk}(u, v)$, is empty of any other points of $S$ inside. An edge is in RNG if and only if the lune defined by this edge is empty. The lune defined by edge $u v$, denoted by lune $(u, v)$, is the intersection of two disks centered at $u$ and $v$ with radius $\|u v\|$. Obviously, RNG is a subgraph of GG, which is a subgraph of the Delaunay triangulation. Since Delaunay triangulation is planar, all these three structures are planar and have at most $O(n)$ edges.

All these structures are defined solely on the given point set and can be viewed as defined on the complete geometric graph topology. Recently, Li et al. [9], motivated by constructing distributed protocols for network routing in mobile networks, extended these definitions to account for the edge structures in the unit disk graph. The unit disk graph is used for topology control and power efficient topology construction for wireless ad hoc networks. In wireless ad hoc networks, nodes can directly communicate with all nodes within its transmission range, which is often normalized to one unit. For a unit disk graph $G$, [9] defined the $k$-localized Delaunay graph as follows. A triangle $\triangle u v w$ formed by edges in $G$ is a $k$-localized Delaunay triangle if its circumcircle is empty of nodes which are within $k$ hops of $u$, or $v$, or $w$. The $k$-localized Delaunay graph $L D e l^{k}$ contains all $k$-localized Delaunay triangles and all Gabriel graph edges on $G$. In [9] it is shown that $L D e l^{k}$ is a planar graph for $k \geq 2$ and $L D e l^{1}$ has thickness 2 . 
However, graphs representing communication links are rarely so completely specified as the unit disk graph. We thus consider the general structure of arbitrary graphs defined by points in the plane, geometric graphs, i.e., its edges are straightline segment connecting the endpoints. For example, for wireless communications, different nodes may have different transmission radius. Consequently, two nodes can communicate directly if they are within the transmission range of each other, i.e., there is a communication link between these two nodes. The graph formed by all such communication links is different from the traditional disk graph, in which two nodes are connected by a straight edge if the two corresponding disks centered at these two nodes intersect. And for wireless communications, two nodes sometimes cannot communicate directly even though they are within the transmission range of each other, due to the blocking of the signal by some barrier. As another example, paths may be required to be found in visibility graphs defined amongst polygonal obstacles in the plane. Traditional proximity structures are often defined based solely on the information of points. We consider the effect on these proximity structures biased by the changed neighborhood created by the topology of geometric graphs. The use of these proximity structures to reduce the complexity of the underlying graph while still retaining connectivity or path properties of the original graph is an interesting issue for research.

In this paper we first present several new proximity structures, based on a given geometric graph $G=(V, E)$. We show relationships between these structures and bounds on their sizes. Most of our definitions are for undirected graph, but can be extended to directed graphs also. Let $N_{G}^{k}(u)$ be all nodes that are within $k$ hops of node $u$ in $G$. We define the zero-edge oriented localized Delaunay graph on graph $G$, denoted by $\operatorname{LDel}_{0}(G)$. This consists of all edges $u v \in E$ such that there is a circle passing through $u$ and $v$, which contains no other point $w$ inside the circle. The one-edge oriented $k$-localized Delaunay graph on $G$, denoted by $\operatorname{LDel}_{1}^{k}(G)$, consists of all edges $u v \in E$ such that there is a circle passing through $u$ and $v$, which contains no point $w \in N_{G}^{k}(u) \cup N_{G}^{k}(v)$ inside. Finally, the two-edge oriented $k$-localized Delaunay neighborhood graph on $G$, denoted by $L D e l_{2}^{k}(G)$, consists of all edges $u v \in E$ such that there is a circle passing through $u$ and $v$, which contains no point $w \in N_{G}^{k}(u) \cap N_{G}^{k}(v)$ inside.

These definitions are extended in the natural way to Gabriel Graphs and the relative neighborhood graphs. Define the $k$-localized Voronoi region of a vertex $v$ as the set of points $p$ such that $v$ is the closest vertex to $p$ among $v$ and all nodes $w$ such that $w \in N_{G}^{k}(u)$. The union of all such region is called the one-edge oriented $k$-localized Voronoi diagram, denoted by $L V \operatorname{or}_{G}^{k}(V)$. We show that the localized Voronoi diagram and Delaunay triangulation are dual of each other: given an edge $u v \in G, u v$ is in the one-edge $k$-localized Delaunay triangulation iff their corresponding Voronoi regions in $k$-localized Voronoi diagram share a common boundary.

We study the edge complexity of the proximity diagrams. Given a geometric graph $G$, we show that the one-edge oriented Delaunay graph, $\operatorname{LDel}_{1}^{k}(G)$ has at most $O\left(n^{5 / 3}\right)$ edges; and the one-edge oriented Gabriel graph has at most 
$O\left(n^{3 / 2}\right)$ edges. Notice that the zero-edge oriented structures defined so far always have at most $O(n)$ edges due to the planar property. However, the two-edge oriented structures could have $O\left(n^{2}\right)$ edges. When the graph $G$ is the communication graph $M G$ derived from the wireless networks, we show that the two-edge oriented Gabriel graph has at most $O\left(n^{5 / 3} \log \frac{r_{\max }}{r_{\min }}\right)$ edges, where $r_{\max }$ and $r_{\min }$ are the maximum and minimum transmission range respectively. In addition, we show that all one-edge oriented localized structures on MG have thickness $1+2 \log _{2} \frac{r_{\max }}{r_{\min }}$. We also study some conditions under which the proposed structures are planar graphs.

The remaining of the paper is organized as follows. We define the generalized Delaunay triangulation and Voronoi diagram on general geometry graphs and study their duality and the edge complexity in Section 2. We further extend this ideas to the relative neighborhood graph, and Gabriel graph in Section 3. We study their properties when the geometry graph is derived from wireless communications in Section 4. We conclude our paper in Section 5.

\section{Generalized Delaunay Triangulation, Voronoi Diagram}

Voronoi diagram and Delaunay triangulation have been widely used in many areas. A triangulation of $V$ is a Delaunay triangulation, denoted by $\operatorname{Del}(V)$, if the circumcircle of each of its triangles does not contain any other vertices of $V$ in its interior. The Voronoi region, denoted by $\operatorname{Vor}(p)$, of a vertex $p$ in $V$ is a collection of two dimensional points such that every point is closer to $p$ than to any other vertex of $V$. The Voronoi diagram for $V$ is the union of all Voronoi regions $\operatorname{Vor}(p)$, where $p \in V$. The Delaunay triangulation $\operatorname{Del}(V)$ is also the dual of the Voronoi diagram: two vertices $p$ and $q$ are connected in $\operatorname{Del}(V)$ if and only if $\operatorname{Vor}(p)$ and $\operatorname{Vor}(q)$ share a common boundary.

\subsection{Definitions}

In this section, we extend the Voronoi region and the Delaunay triangulation from being defined on a point set to being defined on a geometric graph.

The zero-edge oriented localized Delaunay graph on a geometry graph $G=$ $(V, E)$, denoted by $L D e l_{0}(G)$, consists of all edges $u v \in E$ such that there is a circle passing through $u$ and $v$, containing no other point $w$ inside the circle. Obviously, $L \operatorname{Del}_{0}(G)=\operatorname{Del} \cap G$. The one-edge oriented $k$-localized Delaunay graph on $G$, denoted by $L D e l_{1}^{k}(G)$, consists of all edges $u v \in E$ such that there is a circle passing through $u$ and $v$, which contains no point $w \in N_{G}^{k}(u) \cup N_{G}^{k}(v)$ inside. The two-edge oriented $k$-localized Delaunay neighborhood graph on $G$, denoted by $L D e l_{2}^{k}(G)$, consists of all edges $u v \in E$ such that there is a circle passing through $u$ and $v$, containing no point $w \in N_{G}^{k}(u) \cap N_{G}^{k}(v)$ inside. Notice $L D e l_{i}^{k+1}(G) \subseteq L D e l_{i}^{k}(G)$ for $i=1,2$, and $L D e l_{0}(G) \subseteq L D e l_{1}^{k}(G) \subseteq L G G_{2}^{k}(G)$.

Let line $l_{v w}$ be the perpendicular bisector of segment $v w$ and let $h_{v w}$ denote the half-space partitioned by $l_{v w}$, containing the vertex $v$. Then it is well-known 
that the Voronoi region $\operatorname{Vor}(v)=\bigcap_{w \in V} h_{v w}=\bigcap_{v w \in \operatorname{Del}(V)} h_{v w}$. Given a geometry graph $G$, the $k$-localized Voronoi region of a vertex $v \in V$, denoted by $L \operatorname{Vor}_{G}^{k}(v)$, is the intersection of all half-spaces $h_{v w}$ such that $w \in N_{G}^{k}(v)$, i.e.,

$$
L V \operatorname{or}_{G}^{k}(v)=\bigcap_{w \in N_{G}^{k}(v)} h_{v w}=\left\{x \mid\|x-v\| \leq\|x-w\|, \forall w \in N_{G}^{k}(v)\right\} .
$$

\subsection{Duality}

Let $\gamma$ be a function mapping every vertex of $V$ to a polygonal region, which could be unbounded; $\delta$ be some simple graph on $V$. Then functions $\gamma$ and $\delta$ are $d u a l$ of each other, denoted by $\gamma \perp \delta$, if we have: given any edge $u v \in G, \gamma(u)$ and $\gamma(v)$ share a common boundary segment iff vertices $u$ and $v$ are connected in $\delta$. It is well-known that $V o r \perp D e l$ for any point set $V$.

Theorem 1. For any geometry graph $G, L \operatorname{Lor}_{G}^{k} \perp \operatorname{LDel}_{1}^{k}(G)$.

Proof. Given any edge $u v \in G$, if $L V \operatorname{Lr}_{G}^{k}(u)$ and $L V o r_{G}^{k}(v)$ share some common boundary segment then the shared common boundary must be on the perpendicular bisector $l_{u v}$ of segment $u v$. Figure 1 (a) illustrates the proof that follows. Consider any point $x$ on the shared segment of $L \operatorname{Vor}_{G}^{k}(u)$ and $L \operatorname{Vor}_{G}^{k}(v)$. For any vertex $w \in N_{G}^{k}(u),\|x-u\| \leq\|x-w\|$. It implies that $w$ is not inside the disk centered at $x$ with radius $\|x-u\|$. Similarly, for any vertex $y \in N_{G}^{k}(v)$, $\|x-v\| \leq\|x-y\|$. It implies that $y$ is not inside the disk centered at $x$ with radius $\|x-v\|=\|x-u\|$. Therefore, there is a disk (centered at $x$ ) passing through vertices $u, v$ that does not contain any vertex from $N_{G}^{k}(u) \cup N_{G}^{k}(v)$ inside. Thus, $u v \in L \operatorname{Del}_{1}^{k}(G)$.

Consider any edge $u v$ from $L D e l_{1}^{k}(G)$. Then there is a disk passing through $u, v$ that is empty of $N_{G}^{k}(u) \cup N_{G}^{k}(v)$. Let $B(x,\|x-u\|)$ be such disk. Then for any $w \in N_{G}^{k}(v)$, we have $\|x-u\| \leq\|x-w\|$. It implies that $x \in \operatorname{LVor}_{G}^{k}(u)$. Similarly, $x \in L \operatorname{Vor}_{G}^{k}(v)$. Due to the presence of the edge $u v$ in $G$, we know that $L V \operatorname{ror}_{G}^{k}(u)$ and $L V \operatorname{or}_{G}^{k}(v)$ are on different sides of the bisector $l_{u v}$. By definition of the one-edge localized Voronoi region, we know that $L \operatorname{Vor}_{G}^{k}(u)$ and $L \operatorname{Vor}_{G}^{k}(v)$ share a common boundary segment containing point $x$.

\subsection{Edge Complexity}

It is well-known that the Delaunay triangulation has at most $3 n-6$ edges for a two-dimensional point set from its planarity. Thus, all structures that are zero-edge oriented have at most $O(n)$ edges. However, it is easy to construct a geometry graph such that all other structures introduced so far are not planar graphs. Thus, it is not obvious how many edges each of these new structures have. Recently, there had been some studies on the complexity of these geometry structures on unit disk graphs. Li et al. [9] proved that the (one-edge oriented) local Delaunay triangulation on the unit disk graph has $O(n)$ edges. In this 
section, we will further the study of the complexity of these structures when a more general geometry graph $G$ is given.

We first give an upper bound on the number of edges of $L D e l_{1}^{k}(G)$ on a general geometry graph $G$. To do so, we first review the following theorem proved in [10] (Theorem 11 from Chapter 4).

Theorem 2. [10] $A K_{s, t}$-free graph $G$ with $n$ vertices has size at most

$$
\frac{1}{2}(s-1)^{1 / t} n^{2-1 / t}+\frac{1}{2}(t-1) n
$$

Theorem 3. Graph $L D e l_{1}^{k}(G)$ has no more than $O\left(n^{\frac{5}{3}}\right)$ edges.

Proof. We prove that $\operatorname{LDel}_{1}^{k}(G)$ has no $K_{3,3}$ subgraph. For the sake of contradiction, assume that $L D e l_{1}^{k}(G)$ has a $K_{3,3}$ subgraph composed of six vertices $u_{1}$, $u_{2}, u_{3}, v_{1}, v_{2}$, and $v_{3}$. Nodes $u_{i}$ and $v_{j}$ are connected for $i=1,2,3$ and $j=1,2,3$. Notice that the subgraph $K_{3,3}$ is not a planar graph. Without loss of generality, we assume that edges $u_{1} v_{2}$ and $u_{2} v_{1}$ intersect. Then $u_{1}, u_{2}, v_{1}$, and $v_{2}$ form a convex hull $u_{1} u_{2} v_{2} v_{1}$. Notice that we have assumed that there are no four vertices co-circular. ¿From the pigeonhole principal, either $\angle u_{1} u_{2} v_{2}+\angle u_{1} v_{1} v_{2}>\pi$ or $\angle u_{2} v_{2} v_{1}+\angle u_{2} u_{1} v_{1}>\pi$. Assume that $\angle u_{1} u_{2} v_{2}+\angle u_{1} v_{1} v_{2}>\pi$. Then any circle passing through $u_{1}$ and $v_{2}$ either contains $u_{2}$ or $v_{1}$. It is a contradiction to the existence of edge $u_{2} v_{2}$ in $L D e l_{1}^{k}(G)$. ¿From Theorem $2, L D e l_{1}^{k}(G)$ has no more than $2^{-2 / 3} n^{5 / 3}+n=O\left(n^{\frac{5}{3}}\right)$ edges.

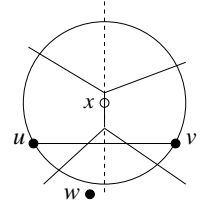

(a)

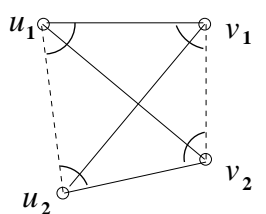

(b)

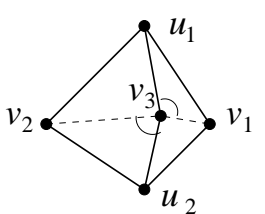

(c)

Fig. 1. (a): $L D e l_{1}^{k}(G)$ and $L V o r_{G}^{k}$ are dual. (b): No subgraph $k_{2,2}$ with crossing edges exists in $L D e l_{1}^{1}(G)$. (c): $L G G_{1}(G)$ does not have $K_{2,3}$ subgraph.

The above theorem is true only if the points are in a general position, i.e., no four points are co-circular. The proof of the above theorem implies that $\operatorname{LDel}_{1}^{k}(G)$ does not contain the structure of a crossing $C_{4}$ as a subgraph. Generally, we would like to know what is the tight upper bound on the number of edges any geometry graph that is free of a crossing $C_{4}$. The above theorem implies that there are at most $O\left(n^{\frac{5}{3}}\right)$ edges. Out conjecture is that there is only $O(n)$ edges. Notice that the two-edge oriented $k$-localized structure could have $O\left(n^{2}\right)$ edges, e.g., when $G$ is a bipartite graph. 


\section{Geometric RNG and GG}

We next extend this idea to the relative neighborhood graph and the Gabriel graph to any geometry graph.

\subsection{Definitions}

The zero-edge oriented localized relative neighborhood graph on a geometry graph $G=(V, E)$, denoted by $L R N G_{0}(G)$, consists of all edges $u v \in E$ such that there is no point $w$ inside lune $(u, v)$. The one-edge oriented $k$-localized relative neighborhood graph on graph $G$, denoted by $L R N G_{1}^{k}(G)$, consists of all edges $u v \in E$ such that there is no point $w \in N_{G}^{k}(u) \cup N_{G}^{k}(v)$ inside lune $(u, v)$. The two-edge oriented $k$-localized relative neighborhood graph on graph $G$, denoted by $\operatorname{LRNG}_{2}^{k}(G)$, consists of all edges $u v \in E$ such that there is no point $w \in$ $N_{G}^{k}(u) \cap N_{G}^{k}(v)$ inside lune $(u, v)$.

Obviously, $L R N G_{i}^{k+1}(G) \subseteq L R N G_{i}^{k}(G)$ for $i=1,2$, and $R N G \cap G=$ $L R N G_{0}(G) \subseteq L R N G_{1}^{k}(G) \subseteq L R N G_{2}^{k}(G)$. Similarly, we can define localized Gabriel graphs $L G G_{0}(G), L G G_{1}^{k}(G)$, and $L G G_{2}^{k}(G)$ using $\operatorname{disk}(u, v)$ instead of lune $(u, v)$. Then, $G G \cap G=L G G_{0}(G) \subseteq L G G_{1}^{k}(G) \subseteq L G G_{2}^{k}(G), L G G_{i}^{k+1}(G) \subseteq$ $L G G_{i}^{k}(G)$, and $L R N G_{i}^{k}(G) \subseteq L G G_{i}^{k}(G) \subseteq L \operatorname{Del}_{i}^{k}(G)$ for $i=0,1,2$.

\subsection{Edge Complexity}

Theorem 3 implies that graphs $L G G_{1}^{k}(G)$ and $L R N G_{1}^{k}(G)$ also have no more than $O\left(n^{\frac{5}{3}}\right)$ edges due to $L R N G_{1}^{k}(G) \subseteq L G G_{1}^{k}(G) \subseteq L D e l_{1}^{k}(G)$. We have

Theorem 4. Graph $L G G_{1}^{k}(G)$ has at most $O\left(n^{\frac{3}{2}}\right)$ edges.

Proof. We first prove that $L G G_{1}^{k}(G)$ has no $K_{2,3}$ subgraph. Assume that $L G G_{1}^{k}(G)$ has a $K_{2,3}$ subgraph composed of five vertices $u_{1}, u_{2}, v_{1}, v_{2}$, and $v_{3}$. Nodes $u_{i}$ and $v_{j}$ are connected for $i=1,2$ and $j=1,2,3$. Then similar to Theorem 3 , we know that there are no intersections among these edges. It implies that four vertices $u_{1}, u_{2}, v_{1}$, and $v_{2}$ form a convex hull $u_{1} v_{1} u_{2} v_{2}$. There are two cases: node $v_{3}$ is inside the convex hull; it is outside of the convex hull. When node $v_{3}$ is outside of the convex hull, we can rename the vertices. Thus, generally, we can assume that node $v_{3}$ is inside the convex hull $u_{1} v_{1} u_{2} v_{2}$. See Figure 1 . Then one of the angles among $\angle u_{1} v_{3} v_{2}, \angle u_{2} v_{3} v_{2}, \angle u_{2} v_{3} v_{1}$, and $\angle u_{1} v_{3} v_{1}$ is at least $\pi / 2$. It implies that one of the disks using $u_{1} v_{1}, v_{1} u_{2}, u_{2} v_{2}$, or $v_{2} u_{1}$ as diameter contains node $v_{3}$. It is a contradiction to their existence in $L G G_{1}^{k}(G)$.

It was shown that a graph without a $K_{r, s}$ subgraph has edges at most $n^{2-\frac{1}{r}}$ where $r \leq s$. Thus, $L G G_{1}^{k}(G)$ has at most $\frac{\sqrt{2}}{2} n^{3 / 2}+n / 2=O\left(n^{\frac{3}{2}}\right)$ edges.

The proof of the upper bounds of the number of edges in local Delaunay triangulation and other relatives is based on the general graph structure. We expect a tight bound by using more geometric properties of the structures. 


\subsection{Planarity}

It was proved that $R N G(V), G G(V)$, and $\operatorname{Del}(V)$ are planar graphs. Li et al. [9] recently showed that $L D e l_{1}(G)^{1}$ on UDG is not a planar graph, but $L D e l_{1}^{k}(G)$ on UDG is always a planar graph for any integer $k>1$. The following lemma presents a sufficient condition such that all localized structures $L D e l_{1}^{k}(G)$ are planar graphs for any integer $k>1$.

Lemma 1. Assume that the geometry graph $G$ is such that, given any two intersected edges uv and $x y$, at least one of the four edges of the convex hull of $u$, $v, x$, and $y$ is in $G$. Then all localized structures $L R N G_{1}^{k}(G), L G G_{1}^{k}(G)$, and $L D e l_{1}^{k}(G)$ are planar graphs for any integer $k>1$.

Proof. We only have to prove that $L D e l_{1}^{k}(G)$ is a planar graph if $G$ satisfies the condition and $k>1$. Consider any two intersected edges $u v$ and $x y$. Without loss of generality, assume that four vertices $u, x, v$, and $y$ are placed clockwise and the edge $u x \in G$. See Figure 2 (a) for an illustration.

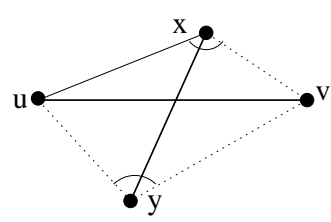

(a)

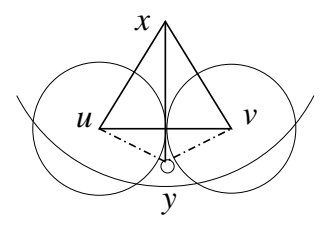

(b)

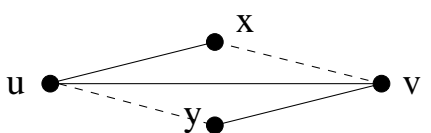

(c)

Fig. 2. (a): Either $x y$ or $u v$ does not belong to $L D e l_{1}^{k}(G)$, for $k \geq 2$. (b): $L G G_{1}^{1}(D G)$ and $L D e l_{1}^{1}(D G)$ are not planar graphs. (c): Here $\angle u x v+\angle u y v>\pi$.

¿From the pigeonhole principle, either $\angle u x v+\angle v y u \geq \pi$ or $\angle y u x+\angle y v x \geq \pi$. Assume that $\angle u x v+\angle v y u \geq \pi$. Then any circle passing through edge $u v$ must contain $x$ or $y$ or both. Notice that both $x$ and $y$ are from $N_{G}^{2}(u)$. It implies that edge $u v$ cannot be in $\operatorname{LDel}_{1}^{k}(G)$ for any $k>1$.

The condition specified in Lemma 1 is satisfied by most practical geometry graphs such as the unit disk graph, the disk graph. Here a graph $G=(V, E)$ is disk graph, denoted by $D G$, if there is a two-dimensional disk $d(u)$ (with radius $r_{u}$ ) for each vertex $u$ such that an edge $u v \in E$ iff $d(u)$ and $d(v)$ intersect.

Theorem 5. $L R N G_{1}^{k}(D G), L G G_{1}^{k}(D G)$, and $L D e l_{1}^{k}(D G)$ are planar, $\forall k>1$.

Proof. Given a disk graph $D G$, assume that we have two intersected edges $u v$ and $x y$. See Figure 2 (a) for an illustration. We will show that one of the edges on the convex hull exists in the disk graph.

For the sake of contradiction, assume that all four edges are not in the disk graph. Then $\|u x\|>r_{u}+r_{x},\|x v\|>r_{v}+r_{x},\|v y\|>r_{v}+r_{y}$, and $\|u y\|>r_{u}+r_{y}$. ¿From triangle inequality, $\|u x\|+\|v y\|<\|u v\|+\|x y\|,\|u y\|+\|v x\|<\|u v\|+\|x y\|$. 
Thus, $\|u v\|+\|x y\|>r_{u}+r_{v}+r_{x}+r_{y}$. The existences of edges $u v$ and $x y$ imply that $\|u v\| \leq r_{u}+r_{v}$, and $\|x y\| \leq r_{x}+r_{y}$, which contradicts the previous bound. Thus, one of the four edges is in $G$ if $G$ is a disk graph, which, together with lemma 1 finishes the proof.

Figure 2 (b) gives an example such that structures $L G G_{1}^{1}(D G)$, and $L D e l_{1}^{1}(D G)$ are not planar graphs. Here node $x$ has the largest disk and node $y$ has the smallest and $\pi / 3<\angle x u y=\angle x v y<\pi / 2$, and $\angle u x v<\pi / 3$. Thus, edges $x u, x v, x y$ and $u v$ are preserved in $L G G_{1}^{1}(D G)$ and $L D e l_{1}^{1}(D G)$.

Theorem 6. $L R N G_{1}^{1}(D G)$ is planar.

Proof. Assume that there are two intersected edges $x y$ and $u v$ in $L R N G_{1}^{k}(D G)$. Similar to the proofs in Theorem 5, we can actually show that there are two adjacent edges of the convex hull uxvy existing in the disk graph. W.l.o.g, assume that $x u$ and $x v$ are in the disk graph. If $\angle u x v>\pi / 3$, edge $u v$ cannot belong to $L R N G_{1}^{k}(D G)$. Otherwise, one of the angles $\angle x u v$ and $\angle x v u$ is larger than $\pi / 3$, which implies that edge $x y$ cannot belong to $L R N G_{1}^{k}(D G)$. We have contradictions in both cases. Thus, no edges intersect in $L R N G_{1}^{k}(D G)$.

Notice that, the conditions specified in Lemma 1 are not satisfied by some other interesting geometry graphs, such as mutually-inclusion communication graph defined later for wireless ad hoc networks.

\subsection{Minimum Spanning Tree}

Unfortunately, the zero-edge oriented or one-edge oriented localized structures may be disconnected. The right figure of Figure 2 illustrates such an example, in which edge $u v$ is removed in any zero-edge or one-edge oriented localized structures. Therefore, they do not always contain the minimum spanning tree of graph $G$.

Lemma 2. Assume that, given any edge uv, the lune $(u, v)$ is either empty of $N_{G}^{1}(u) \cup N_{G}^{1}(v)$ or it contains a vertex $w$ such that $w u$ and $w v$ are edges of $G$, then $M S T_{G}(V) \subseteq L R N G_{1}^{1}(G)$.

Assume that, given any edge uv, either (1) disk $(u, v)$ is empty of $N_{G}^{1}(u) \cup$ $N_{G}^{1}(v)$ or (2) lune $(u, v)$ contains a vertex $w$ such that $w u$ and $w v$ are edges of $G$. Then $M S T_{G}(V) \subseteq G G_{1}^{1}(G)$.

Assume that, given any edge uv, either (1) there is a disk passing through uv and empty of $N_{G}^{1}(u) \cup N_{G}^{1}(v)$ or (2) lune $(u, v)$ contains a vertex $w$ such that $w u$ and $w v$ are edges of $G$. Then $M S T_{G}(V) \subseteq L D e l_{1}^{1}(G)$.

The proof is simple and omitted. Similarly, it is easy to show that all two-edge oriented $k$-localized structures do contain the Euclidean minimum spanning tree as a subgraph. As we will show later that, these structures have sub-quadratic number of edges for some special communication graphs derived from wireless as hoc networks. This makes a fast distributed computation of the minimum 
spanning tree possible. Notice that, it is well-known [11] that the optimal time and communication complexity of computing $M S T_{G}$ in a distributed manner is proportional to $O(n)$ and $O(m+n \log n)$ respectively.

\section{Structures on Graphs from Wireless Ad Hoc Networks}

In wireless ad hoc networks, there are some special geometry graphs. Consider a set of wireless device distributed in a two-dimensional plane. Assume each point $u$ has a fixed transmission range $r_{u}$. A mutual inclusion graph, denoted by $M G$ hereafter, used for ack-based communication in wireless ad hoc networks, has an edge $u v$ if and only if $\|u v\| \leq \min \left(r_{u}, r_{v}\right)$. In [9], Li et al. showed that the oneedge oriented $k$-Localized Delaunay graph $L D e l_{1, U D G}^{k}$ has only a linear number of edges. Moreover, they showed that it can be constructed using only $O(n)$ total messages in wireless ad hoc communications model, i.e., assuming that a message sent by a node can be received by all nodes within its transmission range.

\subsection{Complexity of $L R N G_{1}^{k}(M G), L G G_{1}^{k}(M G), L D e l_{1}^{k}(M G)$}

For simplicity, we first study their complexities when the transmission radius of all nodes is within a constant factor of each other. Since for general graph $G$, the one-edge oriented localized Gabriel graph has at most $O\left(n^{3 / 2}\right)$ edges, thus all structures $L R N G_{1}^{k}(M G)$ and $L G G_{1}^{k}(M G)$ have also at most $O\left(n^{3 / 2}\right)$ edges. Additionally, $L D e l_{1}^{k}(M G)$ has at most $O\left(n^{5 / 3}\right)$ edges.

Here we will show a stronger result. Let $r_{\min }$ be the smallest transmission range; $r_{\max }$ be the maximum transmission range of all nodes.

Theorem 7. The structure $L G G_{1}^{k}(M G)$ has thickness 2 if $r_{\max } \leq \sqrt{2} r_{\min }$.

Proof. First of all, it is easy to show that all edges with length at most $r_{\text {min }}$ belongs to the Gabriel graph of the unit disk graph defined over all nodes with transmission range $r_{\min }$. Thus, the number of all such edges is at most $3 n-6$ since the Gabriel graph over any unit disk graph is planar. We then show that the number of edges with length larger than $r_{\min }$ also forms a planar graph.

Assume, for contradiction, there are two edges $u v$ and $x y$ that intersect. Here $r_{\min }<\|u v\| \leq r_{\max } \leq \sqrt{2} r_{\min }$, so does $\|x y\|$. See Figure 2 (a) for illustration. We then show that one of the four edges of $x u, u y, y v$ and $v x$ has length at most $r_{\min }$. Assume that all such four edges have length larger than $r_{\min }$. W.l.o.g, assume that $\angle u x v+\angle u y v \geq \pi$ and the angle $\angle u x v \geq \pi / 2$. Then $\|u v\|^{2}=$ $\|u x\|^{2}+\|x v\|^{2}-2\|u x\| \cdot\|x v\| \cdot \cos (\angle u x v)>2 r_{\min }^{2}$. Thus $\|u v\|>\sqrt{2} r_{\min }$, which is a contradiction. Thus, we know that one of the two edges $u x$ and $x v$ has length at most $r_{\min }$. Assume that $\|u x\| \leq r_{\min }$. Thus link $u x$ belongs to the original communication graph. Consequently, in the original communication graph, node $x$ is inside $\operatorname{disk}(u, v)$ and has an edge $x u$ to node $u$, which is a contradiction to the existence of edge $u v$ in graph $L G G_{1}^{k}(G)$.

Since $L G G_{1}^{k}(M G)$ contains $L R N G_{1}^{k}(M G)$ as a subgraph, graph $L R N G_{1}^{k}(M G)$ also has thickness 2 when $r_{\max } \leq \sqrt{2} r_{\min }$. Li et al. [9] proved that the localized 
Delaunay triangulation $L D e l_{1}^{k}(G)$ is a planar graph if $G$ is a unit disk graph and $k \geq 2$. Similarly, we have

Theorem 8. If $k \geq 2$ and $r_{\max } \leq \sqrt{2} r_{\min }$, then LDel ${ }_{1}^{k}(M G)$ has thickness 2 .

By a simple bucketing of the edges into the following buckets: $\left(0, r_{\min }\right]$, $\left(r_{\min }, \sqrt{2} r_{\text {min }}\right], \cdots,\left(\sqrt{2}^{i} r_{\min }, \sqrt{2}^{i+1} r_{\min }\right], \cdots,\left(\sqrt{2}^{t-1} r_{\min }, \sqrt{2}^{t} r_{\min }\right]$, it is easy to prove the following theorem. Here $\sqrt{2}^{t} r_{\min } \geq r_{\max }$ and $\sqrt{2}^{t-1} r_{\min }<r_{\max }$.

Theorem 9. Let $\beta=r_{\max } / r_{\min }$. Then $L R N G_{1}^{k}(M G)$ and $L G G_{1}^{k}(M G)$ have thickness $1+2 \log _{2} \beta$ and LDelk $(M G)$ has thickness $1+2 \log _{2} \beta$, if $k \geq 2$.

\subsection{Complexity of $L R N G_{2}^{k}(M G), L G G_{2}^{k}(M G)$, and $L D e l_{2}^{k}(M G)$}

We study the structure $L G G_{2}(M G)$ when the transmission radius of all nodes is within a constant factor of each other. Assume the minimum transmission range is $r$ and the maximum transmission range is $\beta r$, where $\beta$ is a constant.

First of all, all edges in $L G G_{2}^{k}(M G)$ with length at most $r$ form a planar graph since they are in the Gabriel graph over a unit disk graph (each node with transmission range $r$ ). Thus, the number of edges with length at most $r$ is at most $3 n$. We then study the edges with length larger than $r$ but less than $\beta r$. We prove that the number of edges with length $\in(r, \sqrt{2} r]$ is at most $O\left(n^{5 / 3}\right)$.

Lemma 3. The number of edges in $L G G_{2}^{k}(M G)$ with length between $r$ and $\sqrt{2} r$ is at most $O\left(n^{5 / 3}\right)$, where $G$ is the mutually-inclusion communication graph defined over a set nodes whose transmission radius is at least $r$ and at most $\sqrt{2} r$.

Proof. We prove that the crossing circle $C_{4}$ is a forbidden subgraph. Assume that there is a crossing $C_{4}=x v u y$ formed by crossing edges $u v$ and $x y$. Obviously, all such nodes have transmission range at least $r$.

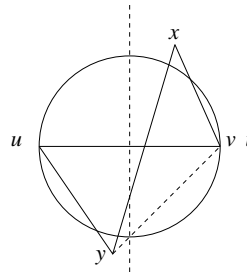

(a)

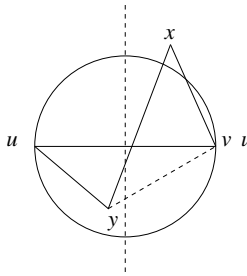

(b)

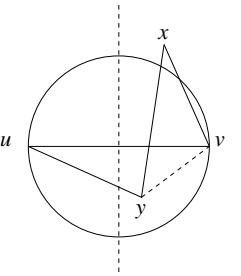

(c)

Fig. 3. Crossing $C_{4}$ is a forbidden subgraph.

We first prove that both $x$ and $y$ cannot be outside of $\operatorname{disk}(u, v)$. Suppose that happens. W.l.o.g., assume that the midpoint of $u v$ is on the same side of $x y$ as $u$ (Figure $4.2(\mathrm{a})$ ). Then $\angle x v y>\pi / 2$. 
For both cases, if $\|v y\| \leq r$, then the edge $v y$ is in the original mutual communication graph since all nodes have transmission range at least $r$. Since $\angle x v y>\pi / 2$ edge $x y$ cannot be in the Gabriel graph. If $\|v y\|>r$, together with $\|x v\| \geq r$ and $\angle x v y>\pi / 2$, we have $\|x y\|>\sqrt{2} r$, which is a contradiction to the fact that we only consider edges with length $\leq \sqrt{2} r$.

Then we know that at least one of $x$ or $y$ or both is inside $\operatorname{disk}(u, v)$. Assume that $y$ is inside. There are two cases here: (b) $y$ is on the same side of bisector of segment $u v$ as $u$; (c) $y$ is on the same side of bisector of segment $u v$ as $v$.

Case (b) is impossible since $\|u y\|<\frac{\sqrt{2}}{2}\|u v\|<\frac{\sqrt{2}}{2} \sqrt{2} r=r$, which is a contradiction to the fact that we only consider edges with length between $r$ and $\sqrt{2} r$.

In case (c), similarly we have $\|v y\|<r$, which implies the existence of edge $v y$ in the original mutual communication graph. This, together with existence of edge $u y$, is a contradiction to the existence of edge $u v$ in the Gabriel graph.

Notice in Theorem 3, we showed that if a graph is $k_{3,3}$ free then it is free of crossing $C_{4}$. This finishes the proof.

By bucketing edges into $1+2 \log _{2} \beta$ buckets, we have

Theorem 10. The number of edges in $L G G_{2}^{k}(G)$ is at most $O\left(n^{5 / 3} \log _{2} \beta\right)$, where $\beta=r_{\max } / r_{\min }$.

Conjecture 1. At most $O(n)$ edges in $L G G_{2}^{k}(M G)$ have length $\in(r, \sqrt{2} r]$.

\section{Conclusion}

In this paper we proposed several new proximity structures on general geometric graphs and studied their complexities for both general geometric graph and some special geometric graphs. We summarize the results about the edge complexities of the structures we have discussed as follows. Here $C_{\beta}=1+2 \log _{2} \beta$ and $\beta=r_{\max } / r_{\min }$. The complexities with a star mark is true only when $k \geq 2$.

Table 1. Upper bounds of the edge numbers.

\begin{tabular}{|l|c|c|c|}
\hline & G & DG & MG \\
\hline \hline$L D e l_{1}^{k}(G)$ & $O\left(n^{5 / 3}\right)$ & $\Theta(n)^{\star}$ & $O\left(C_{\beta} \cdot n\right)^{\star}$ \\
\hline$L D e l_{2}^{k}(G)$ & $\Theta\left(n^{2}\right)$ & & $O\left(C_{\beta} \cdot n^{5 / 3}\right)$ \\
\hline$L G G_{1}^{k}(G)$ & $O\left(n^{3 / 2}\right)$ & $\Theta(n)^{\star}$ & $O\left(C_{\beta} \cdot n\right)$ \\
\hline$L G G_{2}^{k}(G)$ & $\Theta\left(n^{2}\right)$ & & $O\left(C_{\beta} \cdot n^{5 / 3}\right)$ \\
\hline$L R N G_{1}^{k}(G)$ & $O\left(n^{3 / 2}\right)$ & $\Theta(n)$ & $O\left(C_{\beta} \cdot n\right)$ \\
\hline$L R N G_{2}^{k}(G)$ & $\Theta\left(n^{2}\right)$ & & $O\left(C_{\beta} \cdot n^{5 / 3}\right)$ \\
\hline
\end{tabular}

Notice that one way to study the complexity of these geometry structures is from the point view of forbidden subgraphs. Although the complexity of general 
graph with forbidden structure is well-studied, little is known about the complexity of the geometry graph with some forbidden structure. We indirectly showed that any geometry graph on $n$ points with forbidden crossing $C_{4}$ has at most $O\left(n^{5 / 3}\right)$ edges. To the best of our knowledge, this is the currently best known upper bound. However, it is unlikely that we can achieve this upper bound. We summarize some open questions we have discussed in this paper as follows.

1. What are the tight bounds on the sizes of $L D e l_{1}^{k}(G), L D e l_{2}^{k}(G), L G G_{1}^{k}(G)$, $L G G_{2}^{k}(G)$, etc.? We can also consider the case when $G$ is some special graph such as a disk graph $D G$, a mutually-inclusion graph $M G$ etc.

2. What is the size of a geometric graph, free of crossing $C_{4}$. We know that it is at most $O\left(n^{5 / 3}\right)$ for graph of $n$ vertices.

3. How to construct the proximity structures defined in the paper efficiently. For the UDG, Li et al. [9] previously gave an asymptotically optimal method to construct $L D e l_{1}^{k}(U D G)$.

4. Is the graph $L D e l_{2}^{k}(G)$ a spanner? This question would be interest also for some special graphs like the disk graph or the mutually-inclusion graph. Notice that it was known [5] that $G G$ and $R N G$ are not length spanners. Thus localized Gabriel graph and relative neighborhood graphs are not spanners.

\section{References}

1. Edelsbrunner, H.: Algorithms in Combinatorial Geometry. Springer-Verlag (1987)

2. Fortune, S.: Voronoi diagrams and Delaunay triangulations. In: F. K. Hwang and D. -Z. Du, editors, Computing in Euclidean Geometry, World Scientific, (1992) 193-233

3. Preparata, F.P., Shamos, M.I.: Computational Geometry: an Introduction. Springer-Verlag (1985)

4. Gabriel, K., Sokal, R.: A new statistical approach to geographic variation analysis. Systematic Zoology 18 (1969) 259-278

5. Bose, P., Devroye, L., Evans, W., Kirkpatrick, D.: On the spanning ratio of Gabriel graphs and beta-skeletons. In: Proceedings of the Latin American Theoretical Informatics (LATIN). (2002)

6. Jaromczyk, J.W., Kowaluk, M.: Constructing the relative neighborhood graph in three-dimensional euclidean space. Discrete Applied Mathematics (1991) 181-192

7. Jaromczyk, J., Toussaint, G.: Relative neighborhood graphs and their relatives. Proceedings of IEEE 80 (1992) 1502-1517

8. Supowit, K.J.: The relative neighborhood graph, with an application to minimum spanning trees. Journal of Associate Computing Machine (1983)

9. Li, X.Y., Calinescu, G., Wan, P.J.: Distributed construction of planar spanner and routing for ad hoc wireless networks. In: 21st Annual Joint Conference of the IEEE Computer and Communications Societies (INFOCOM). Volume 3. (2002)

10. Bollobás, B.: Extremal Graph Theory. Academic Press (1978)

11. Faloutsos, M., Molle, M.: Creating optimal distributed algorithms for minimum spanning trees. Technical Report CSRI-327 (also in WDAG '95) (1995)

12. Alzoubi, K., Wan, P.J., Frieder, O.: Message-optimal connected-dominating-set construction for routing in mobile ad hoc networks. In: 3rd ACM International Symposium on Mobile Ad Hoc Networking and Computing (MobiHoc'02). (2002) 